\title{
Rapid determination of nanowires electrical properties using a dielectrophoresis-well based system
}

Marios Constantinou, Kai F. Hoettges, Sergiy Krylyuk, Michael B. Katz, Albert Davydov, Grigorios-Panagiotis Rigas, Vlad Stolojan, Michael P. Hughes, and Maxim Shkunov

Citation: Appl. Phys. Lett. 110, 133103 (2017); doi: 10.1063/1.4978930

View online: http://dx.doi.org/10.1063/1.4978930

View Table of Contents: http://aip.scitation.org/toc/apl/110/13

Published by the American Institute of Physics

\section{Articles you may be interested in}

Suppression of conductivity deterioration of copper thin films by coating with atomic-layer materials Appl. Phys. Lett. 110, 131601131601 (2017); 10.1063/1.4979038

Tuning the thickness of exfoliated quasi-two-dimensional $\beta$-Ga2O3 flakes by plasma etching Appl. Phys. Lett. 110, 131901131901 (2017); 10.1063/1.4979028

Current-induced surface roughness reduction in conducting thin films

Appl. Phys. Lett. 110, 103103103103 (2017); 10.1063/1.4977024

Growth of Fe3Si/Ge/Fe3Si trilayers on $\mathrm{GaAs}(001)$ using solid-phase epitaxy

Appl. Phys. Lett. 110, 102103102103 (2017); 10.1063/1.4977833

Quasiparticle self-consistent GW band structure of $\beta$-Ga2O3 and the anisotropy of the absorption onset Appl. Phys. Lett. 110, 132103132103 (2017); 10.1063/1.4978668

Ferroelectric, pyroelectric, and piezoelectric properties of a photovoltaic perovskite oxide Appl. Phys. Lett. 110, 063903063903 (2017); 10.1063/1.4974735
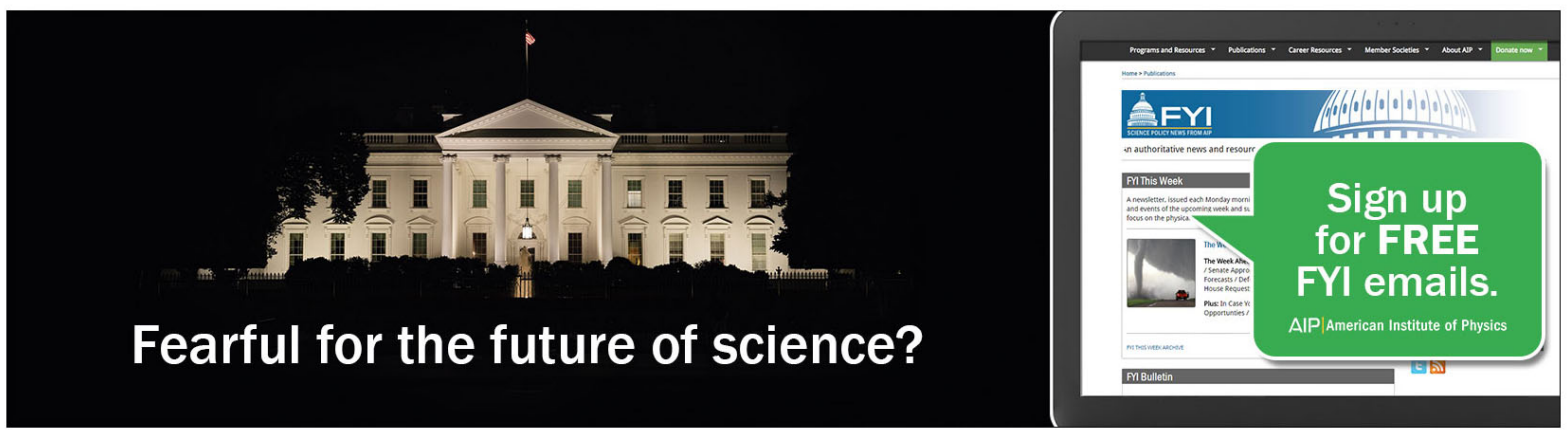


\title{
Rapid determination of nanowires electrical properties using a dielectrophoresis-well based system
}

\author{
Marios Constantinou, ${ }^{1}$ Kai F. Hoettges, ${ }^{2, a)}$ Sergiy Krylyuk, ${ }^{3,4}$ Michael B. Katz, ${ }^{4}$ \\ Albert Davydov, ${ }^{4}$ Grigorios-Panagiotis Rigas, ${ }^{1}$ Vlad Stolojan, ${ }^{1}$ Michael P. Hughes, ${ }^{2, b)}$ \\ and Maxim Shkunov ${ }^{1, b)}$ \\ ${ }^{1}$ Department of Electrical and Electronic Engineering, Advanced Technology Institute, University of Surrey, \\ Guildford, Surrey GU2 7HX, United Kingdom \\ ${ }^{2}$ Centre for Biomedical Engineering, Department of Mechanical Engineering Sciences, University of Surrey, \\ Guildford, Surrey GU2 7HX, United Kingdom \\ ${ }^{3}$ Theiss Research, La Jolla, California 92037, USA \\ ${ }^{4}$ Materials Science and Engineering Division, National Institute of Standards and Technology (NIST), \\ Gaithersburg, Maryland 20899, USA
}

(Received 25 September 2016; accepted 8 March 2017; published online 28 March 2017)

\begin{abstract}
The use of high quality semiconducting nanomaterials for advanced device applications has been hampered by the unavoidable growth variability of electrical properties of one-dimensional nanomaterials, such as nanowires and nanotubes, thus highlighting the need for the characterization of efficient semiconducting nanomaterials. In this study, we demonstrate a low-cost, industrially scalable dielectrophoretic (DEP) nanowire assembly method for the rapid analysis of the electrical properties of inorganic single crystalline nanowires, by identifying key features in the DEP frequency response spectrum from $1 \mathrm{kHz}$ to $20 \mathrm{MHz}$ in just $60 \mathrm{~s}$. Nanowires dispersed in anisole were characterized using a three-dimensional DEP chip (3DEP), and the resultant spectrum demonstrated a sharp change in nanowire response to DEP signal in 1-20 MHz frequency range. The 3DEP analysis, directly confirmed by field-effect transistor data, indicates that nanowires of higher quality are collected at high DEP signal frequency range above $10 \mathrm{MHz}$, whereas lower quality nanowires, with two orders of magnitude lower current per nanowire, are collected at lower DEP signal frequencies. These results show that the 3DEP platform can be used as a very efficient characterization tool of the electrical properties of rod-shaped nanoparticles to enable dielectrophoretic selective deposition of nanomaterials with superior conductivity properties.
\end{abstract} Published by AIP Publishing. [http://dx.doi.org/10.1063/1.4978930]

Semiconducting single-crystalline nanowires (NWs) offer many advantages for the fabrication of solutionprocessed, low-cost printed electronic devices. Their unique characteristics make them potential key building blocks in many emerging applications, such as chemical and biological sensors, ${ }^{1-3}$ high performance field-effect transistors (FETs),${ }^{4-6}$ optical devices, ${ }^{7,8}$ memory elements ${ }^{9-11}$ and energy harvesting nanogenerators. ${ }^{12,13}$

However, NW bottom-up synthesis methods, e.g., vaporliquid-solid (VLS) growth, often produce heterogeneous mixtures of NWs with a range of different electrical properties, and as a consequence, the purification of one-dimensional nanomaterials based on their electrical properties is a significant hurdle for the fabrication of reliable and high-performance devices. Whilst the deposition of NWs into ordered arrays has been demonstrated with various techniques, such as LangmuirBlodgett, ${ }^{14}$ Blown-Bubble films, ${ }^{15}$ flow-directed assembly ${ }^{16}$ and electrostatic interactions, ${ }^{17}$ these techniques do not include a selection step to separate the most desirable NW based on their electrical performance.

We have previously demonstrated the direct selection of supercritical fluid-liquid-solid (SFLS) grown silicon

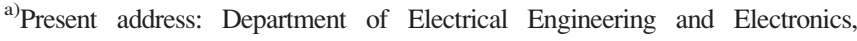
University of Liverpool, Brownlow Hill, Liverpool L69 3GJ, United Kingdom.

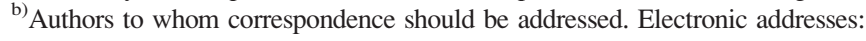
m.shkunov@surrey.ac.uk and m.hughes@surrey.ac.uk
}

nanowires (SiNWs) based on their conduction properties from poly-disperse as-synthesized NWs using dielectrophoresis (DEP) coupled with impedance spectroscopy, highlighting the selective collection of NWs with different electrical properties at various applied frequencies. ${ }^{18}$ DEP is an electrostatic phenomenon of induced motion of a polarizable particle when subjected to an inhomogeneous electric field. Depending on the electrical properties of the particle and suspending medium, the induced DEP force causes the particle to be either attracted towards the region of high electric field gradient (positive DEP) or repelled (negative DEP) along the direction of the field gradient; $;{ }^{19}$ the magnitude and direction of the force is dependent on the electrical properties of particle and medium and on the frequency of the applied field. Consequently, the analysis of the DEP response of nanomaterials over a range of frequencies can be used to determine the properties of suspensions, whilst particles with different properties can be separated by selecting a frequency, where the different populations experience stronger or weaker DEP forces. ${ }^{18}$ The use of DEP characterization has been widely explored in the biosciences but until recently was restricted to laboratories with expertise in the field. However, a new commercial platform that utilizes a disposable, three-dimensional (3D) well electrode chip (3DEP by DEPtech, Uckfield, UK) coupled with a prototype 3DEP system reader (Labtech, Uckfield, UK) makes DEP 
characterization suitable for a wider range of nanomaterials. This 3DEP platform has been used in the measurement of the electrical properties of cells used in cancer studies ${ }^{20-23}$ (including keratinocyte, OOPC and OSCC cells) and drug interaction. ${ }^{24}$ The system is engineered principally for the rapid and near-real-time analysis of the electrical properties of cells, taking typically $10 \mathrm{~s}$ to analyze a population of $c a$. 20000 cells. However, 3DEP system has not been previously used for the characterization of nanomaterials.

Traditionally, DEP electrodes are constructed with dimensions that reflect the size of the particles; however, the 3DEP chip used in this work with electrode gap size of $150 \mu \mathrm{m}$ still provided an efficient characterization tool for $\sim 22 \mu \mathrm{m}$ long SiNWs used in this study.

In this paper, we demonstrate the capability of the 3DEP system as a rapid evaluation tool of the electrical properties of as-synthesized SiNWs ensemble using a frequency spectrum analysis. 3DEP chip frequency response of dispersed SiNWs showed a "threshold" DEP signal frequency of $\approx 1 \mathrm{MHz}$, above which the DEP started to collect higher quality NWs, whereas NWs collected at low DEP signal frequency ranges $(\mathrm{Hz}-\mathrm{kHz})$ were, on average, significantly inferior to high frequency assembled NWs. The whole response spectrum $(1 \mathrm{kHz}-20 \mathrm{MHz})$ measurement of NWs took only $1 \mathrm{~min}$. The direct correlation between DEP signal frequency and the SiNWs' conductivity was confirmed by field-effect transistor (FET) data demonstrating that average current per NW can increase by two order of magnitude from bad to good NWs.

VLS growth of SiNWs was catalyzed using commercially available $60 \mathrm{~nm}$ Au nanoparticles randomly dispersed on poly-L-lysine-functionalized $\mathrm{Si}(111)$ substrates. Growth was performed at $900^{\circ} \mathrm{C}$ and $80 \mathrm{kPa}$ using $30 \mathrm{sccm}$ (standard $\mathrm{cm}^{3} / \mathrm{min}$ ) of $\mathrm{SiCl}_{4}$ and $200 \mathrm{sccm}$ of $\mathrm{H}_{2}$ diluted with $\mathrm{N}_{2}$ to a total flow rate of $1000 \mathrm{sccm}$. Small pieces of Si wafer with asgrown nominally undoped NWs were placed in small vials containing $2 \mathrm{ml}$ of anisole. NWs were dispersed by using ultrasonic agitation at low power $(\approx 200 \mathrm{~W})$ for $10 \mathrm{~s}-15 \mathrm{~s}$. The homogeneous as-grown and dispersed SiNWs exhibited an average $22 \mu \mathrm{m}$ length and $\approx 75 \mathrm{~nm}$ diameter (Fig. 1), as analyzed by scanning electron microscopy (SEM) and transmission electron microscopy (TEM), respectively. TEM studies confirmed the [111] growth direction of NWs. The NWs' diameter was also evaluated and confirmed from the results of atomic force microscopy (AFM) (data not shown).

A DEPtech 3DEP microwell electrode system was used for the determination of the DEP-frequency response of SiNW dispersions. The 3DEP electrode chip contained 20 wells of $1 \mathrm{~mm}$ diameter, each comprising eight gold-plated copper electrodes in a ring configuration separated by polyimide layers, with thicknesses of $70 \mu \mathrm{m}$ of copper and $150 \mu \mathrm{m}$ of polyimide, with a cross-section schematic shown in Fig. 2(a). For each experiment, approximately $75 \mu \mathrm{l}$ of SiNWs dispersed in anisole were injected into the wells of the electrode chip. The principle of operation of the 3DEP is based on the attraction of nanoparticles towards the imbedded electrodes during positive DEP, resulting in a reduction in light scattering, and repulsion from the electrodes during negative DEP, leading to an increased light scattering, when an alternating electric signal is applied to the electrodes. The relative strength of the dielectrophoretic force can be evaluated by measuring the changes of the
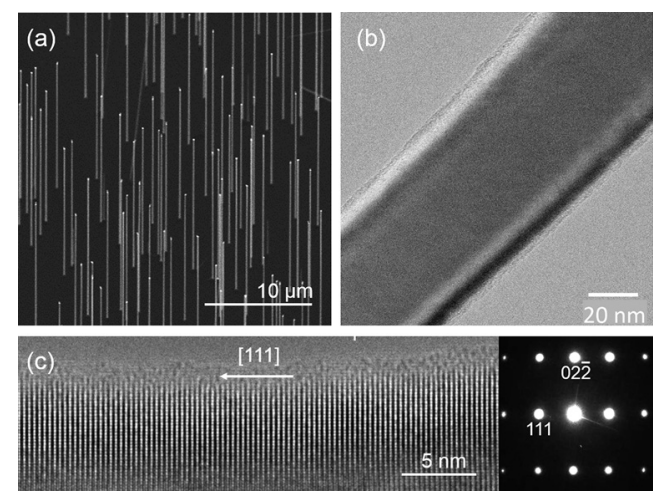

FIG. 1. (a) SEM image (30 tilt) of CVD-grown Si NWs. (b) Low- and (c) high-resolution TEM images of a typical Si NW with $\sim 70 \mathrm{~nm} \mathrm{Si} \mathrm{core} \mathrm{and}$ $\sim 2 \mathrm{~nm}$ thick shell of native oxide. Inset in (c) shows the corresponding selected area electron diffraction pattern.

transmitted light intensity through the well for each applied DEP signal frequency. During the experiment, each of the 20 wells received a different frequency energizing signal in the range $1 \mathrm{kHz}-20 \mathrm{MHz}$ at peak-to peak signal $\left(\mathrm{V}_{\mathrm{pk}-\mathrm{pk}}\right)$ of $10 \mathrm{~V}$. The applied frequency remained constant for each well during the experiment. Wells were excited with $\mathrm{AC}$ signal for $60 \mathrm{~s}$, during which NWs were expected to experience positive DEP, and to move away from the well center. Software was then used to assess the change in NW distribution by measuring the change in light intensity within each well, as the NWs suspended in anisole and reoriented and moved in response to the applied field. The change in light intensity was normalized to intensity of the image of the well captured before applying the electric field, and the relative dielectrophoretic force acting on
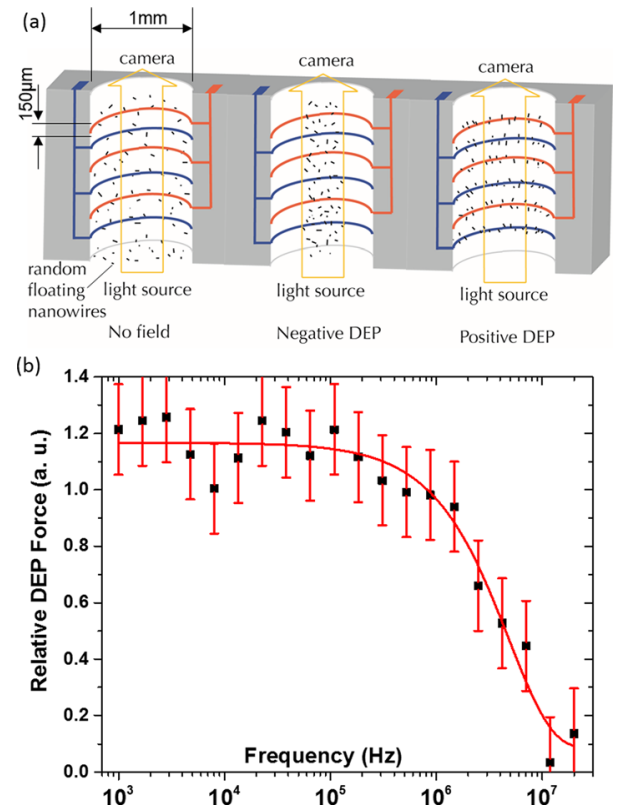

FIG. 2. (a) A cross-section schematic of the 3D well electrode chip used in this work. The nanoparticles can either be attracted towards the electrodes (the walls of the well) by applying a positive DEP or be repelled from the electrodes by applying a negative DEP. (b) Relative DEP force (arbitrary units) as a function of DEP signal frequency for SiNWs suspended in anisole generated by the commercially available 3DEP system reader (3DEP by DEPtech, Uckfield, UK). The points represent the mean DEP response of three independent experiments. The solid red line represents the best fit. Error bars were evaluated from the fluctuations of light intensity due to random movement of NWs in the liquid. 
NWs was then related to the changes of light intensity. When dispersed NWs experience a DEP force in a liquid, they reach their terminal velocity in milliseconds, and so for any observed movement we can assume that velocity is proportional to the DEP force $\left(\mathrm{F}_{\mathrm{DEP}}\right)$ exerted on the particle. $\mathrm{F}_{\mathrm{DEP}}$ for NWs with cylindrical shape is commonly approximated to that of a prolate ellipsoid with major and minor axes equal to the NW length and diameter using the relationship given by Equations (1a) $-(1 \mathrm{c})^{25-30}$

$$
\begin{gathered}
\mathrm{F}_{\mathrm{DEP}}=\frac{2 L \pi r^{2}}{3} \varepsilon_{m} \operatorname{Re}\left\{K_{f}\right\} \nabla E^{2}, \\
K_{f}=\frac{\tilde{\varepsilon}_{p}-\tilde{\varepsilon}_{m}}{\tilde{\varepsilon}_{m}}, \\
\tilde{\varepsilon}_{p}=\varepsilon-j \frac{\sigma_{p}}{\omega}, \quad \tilde{\varepsilon}_{m}=\varepsilon-j \frac{\sigma_{m}}{\omega},
\end{gathered}
$$

where $r$ is NW radius, $L$ is NW length, $\varepsilon_{m}$ is the permittivity of the fluid medium and is the gradient of the electric field strength squared, $K_{f}$ is the Clausius-Mossotti factor and $R e$ denotes "the real part of". $\tilde{\varepsilon}_{p}$ is the permittivity of NW, $\tilde{\varepsilon}_{m}$ is the permittivity of the medium, $\varepsilon_{o}$ is the vacuum permittivity, $\sigma_{p}$ is the conductivity of NW, $\sigma_{m}$ is the conductivity of the medium and $\omega$ is the angular frequency of the electric field.

Examination of Equation (1a) shows that the DEP force scales with particle volume. This means that small-volume nanoparticles typically require larger $\nabla E^{2}$, which is usually obtained by using electrodes with small gap sizes. The electrodes gap in the commercial 3DEP chip is $150 \mu \mathrm{m}$; nevertheless, the nature of semiconducting NWs is that the dipole along the long axis is sufficiently substantial to induce enough DEP force to move the nanoparticles in the suspension medium; consequently, DEP alignment is observed even at distances few tens of microns away from these electrodes.

The dielectrophoretic force spectrum of undoped VLS grown SiNWs obtained by this method is shown in Fig. 2(b). The points represent the mean $(n=3)$ DEP system response at different frequencies; the solid line represents the best fit of the data. The graph shows a plateau response between $1 \mathrm{kHz}$ and $100 \mathrm{kHz}$, followed by a decrease in relative DEP force at around $1 \mathrm{MHz}$ signal frequency. At frequencies $10 \mathrm{MHz}-20 \mathrm{MHz}$, the DEP force reaches lower - but nonzero - values, indicating that a smaller population of NWs still responds to the DEP field.

Whilst the VLS growth of SiNWs could be expected to provide a mono-dispersed collection of nanowires, in terms of both their lengths and their electrical properties, the frequency range over which the DEP dispersion takes place (beginning at $c a .1 \mathrm{kHz}$ and continuing to over $20 \mathrm{MHz}$ ) suggests a range of NW conductivity heterogeneities; the dispersion of electrically homogeneous NWs would typically take one decade to move from $<10 \%$ of the transition to $>90 \% .{ }^{31}$ We note that VLS growth provides SiNWs with highly consistent lengths and diameters, unlike SFLS synthesis we have reported previously, ${ }^{18}$ so in the current study we can disregard the influence of morphological properties such as length and diameter on the DEP response characteristics.

As the frequency increases, the dipole across the nanowires weakens, causing the relative DEP force to drop, so that NWs with the lowest conductivity will exhibit DEP assembly only at low frequencies. The variations in conductivity are expected to originate from the crystal quality of the NWs, such as the concentration of surface and crystalline defects and associated traps. However, at higher frequencies, we predict that $\mathrm{F}_{\mathrm{DEP}}$ will reach a plateau, where highest conductivity NWs can still be collected and then decline close to zero DEP force. For DEP nanowire assembly, this means that SiNWs with various conductive properties will respond to the DEP signal in the range from few $\mathrm{Hz}$ to approximately few hundred kHz, as shown in Fig. 2(b), resulting in a collection of NWs with mediocre conductivity properties, whereas DEP assembly at high frequencies $10-20 \mathrm{MHz}$ will result in the collection of exclusively highest quality and highest conductivity NWs. In relation to other semiconducting NWs and nanomaterials such as groups IV, III-V, and II-VI, we anticipate that the DEP threshold frequency separating the collection of good and bad quality nanoparticles will vary according to their different doping levels and corresponding charge carrier mobilities and will need to be determined experimentally.

The electrical properties of the SiNWs collected at different frequencies were investigated by FET analysis to establish a direct correlation with the effective DEP force spectrum. The FET devices were prepared on $\mathrm{Si} / \mathrm{SiO}_{2}$ substrates, with pre-patterned source-drain electrodes with $10 \mu \mathrm{m}$ gap, where highly doped Si substrate served as the bottom-gate electrode. The dielectrophoretic bottom-contact structures consisting of $2 \mathrm{~nm}$ Ti acting as an adhesion metal followed by $50 \mathrm{~nm}$ gold $(\mathrm{Au})$ or palladium $(\mathrm{Pd})$ layers were patterned by the photolithographic lift-off technique. The choice of the contact metals is dictated by p-type conductivity that is usually observed in undoped SiNWs. ${ }^{32}$ Both Au and Pd metal work functions are closely matched to the valence band edge of Si to enable near-Ohmic contact for p-type transport. ${ }^{33}$ The alignment of the SiNWs was performed using DEP by applying an AC field across two electrodes (at $\mathrm{V}_{\mathrm{pk}-\mathrm{pk}}=12 \mathrm{~V}$ ) forming the source-drain (S/D) contacts of the FET device. The substrate was placed on an inclined surface $\left(\approx 30^{\circ}\right.$ versus the horizontal plane) to allow NW dispersion to flow along the substrate and to cross the dielectrophoretic electrodes, as described previously. ${ }^{18} \mathrm{An}$ example of aligned monolayer of NWs across FET electrodes with $10 \mu \mathrm{m}$ gap is shown in Figs. 3(a) and 3(b). The fabrication of the FET devices was completed by performing a second lift-off process to deposit $80 \mathrm{~nm}$ thick metal contacts on top of the aligned NW for the improvement of the charge carrier injection. Prior to the metal deposition, the edges of the NWs at the S/D contacts regions underwent a diluted hydrofluoric acid treatment for $12 \mathrm{~s}$ to remove the native oxide layer of the NWs, ${ }^{34-36}$ thereby forming a "clean" semiconductorelectrode contact region. The final step included the postanneal of the FET devices at $250^{\circ} \mathrm{C}-300^{\circ} \mathrm{C}$ for $45 \mathrm{~min}$ to improve the metal-semiconductor contact for optimum charge transport characteristics. ${ }^{4}$ A schematic of the bottom-gate FET device with Au contacts is shown in Fig. 4(b). The transistors were characterized using a Keithley 4200 SCS analyzer system in an $\mathrm{N}_{2}$-filled glove box in order to minimize the effects of atmospheric contamination.

Typical transfer characteristics of FETs prepared with NWs aligned at various DEP frequencies are shown in Fig. 

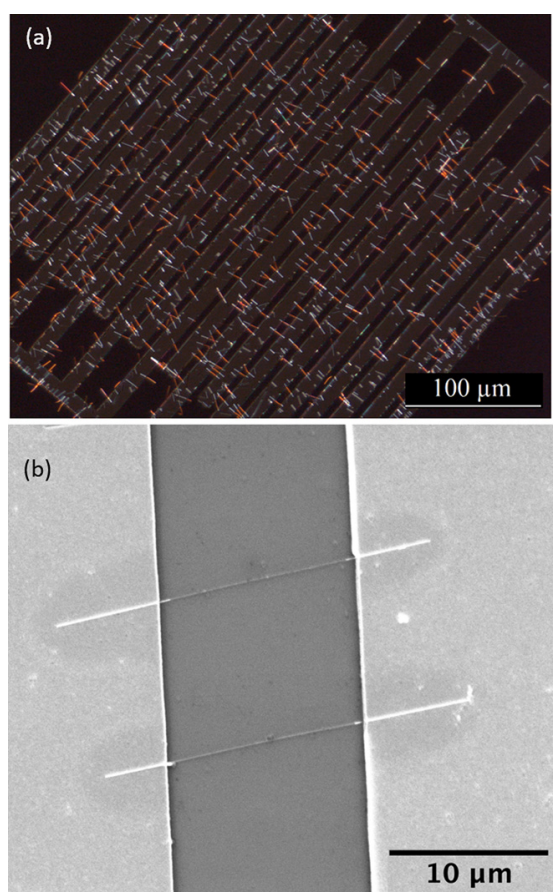

FIG. 3. (a) Polarized microscope image (POM) and (b) SEM image of SiNWs aligned across $10 \mu \mathrm{m}$ FET channel gaps via DEP. POM imaging was used to evaluate the total number of nanowires crossing the FET electrodes.

4(a). The transfer characteristics were normalized to the number of NWs that bridge the S/D electrodes, typically between 50 and 230 nanowires. FET devices with NWs aligned at higher DEP frequencies $(10 \mathrm{MHz}$ and $20 \mathrm{MHz})$ consistently demonstrated higher currents, highlighting nanowires' superior conductivity properties.

In order to evaluate the FET performance, the subthreshold slope (s-s), the trap density $\left(\mathrm{N}_{\text {trap }}\right)$ and mobility were calculated as a function of DEP frequency. The sub-threshold behavior (V/decade) indicates how much gate voltage is needed to turn-on the transistor, and, in addition to the FET geometrical parameters, it also depends on the density of defect/trap states on the NW surface or at the NW/dielectric

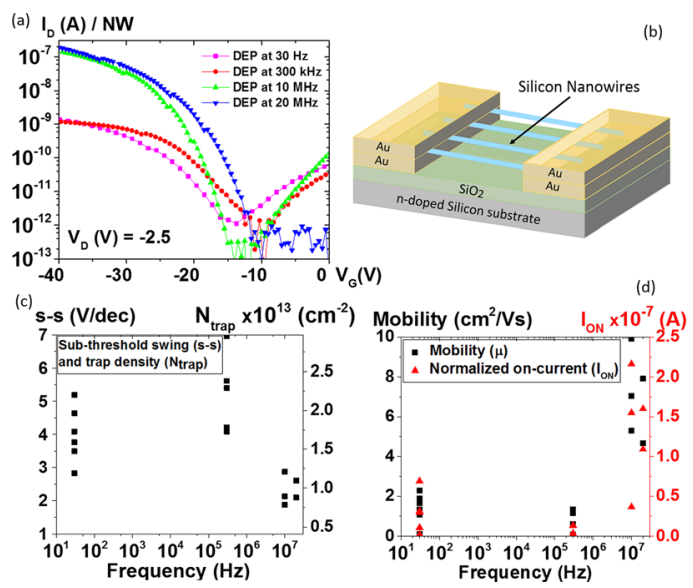

FIG. 4. (a) Transfer characteristic of FETs with NWs aligned via DEP at $30 \mathrm{~Hz}, 300 \mathrm{kHz}, 10 \mathrm{MHz}$ and $20 \mathrm{MHz}$. The plot is normalized to the number of NWs across the FET channel, thus showing an average current per NW. (b) Schematic of a bottom-gate SiNW FET device with Au S/D contacts. (c) and (d) Typical performance data, including sub-threshold swing (s-s), trap density $\left(\mathrm{N}_{\text {trap }}\right)$, mobility $(\mu)$ and normalized on-current $\left(\mathrm{I}_{\mathrm{ON}}\right)$ for a number of FET devices with nanowires aligned at $30 \mathrm{~Hz}, 300 \mathrm{kHz}, 10 \mathrm{MHz}$ and $20 \mathrm{MHz}$. interface. Thus, higher trap density results in less steep current increase and high sub-threshold values. The sub-threshold slope is given by Equations (2) $)^{37,38}$ and (3) $)^{33}$ and is related to the trap density $\left(\mathrm{N}_{\text {trap }}\right)$ according to Equation $(4)^{39,40}$

$$
\begin{gathered}
s-s=\ln (10) \frac{\left(1+\frac{C_{i t}}{C_{b o x}}+\frac{C_{s i}}{C_{b o x}}\right)}{\left(\frac{q}{k T}-\frac{1}{E_{b n w} 2 r}\right)}, \\
s-s=\frac{\Delta V_{G}}{\Delta \log I_{D}}, \\
N_{\text {trap }}=\left[\frac{q(s-s) \log (e)}{k T}-1\right] \frac{C_{N W}}{2 \pi N r L q},
\end{gathered}
$$

where $k$ is the Boltzmann's constant, $T$ is absolute temperature, $q$ is the electron charge, $E_{b n w}$ is the field at the bottom of the $\mathrm{SiNW}, C_{i t}$ is the capacitance of interface states at the $\mathrm{Si}$ oxide-shell/Si region of the NW, $C_{s i}$ is the capacitance of the SiNW, and $C_{b o x}$ is the back oxide capacitance $\left(\mathrm{SiO}_{2}\right)$, $C_{N W}$ is the total gate capacitance, and $N$ is the total number of NWs across the channel.

The carrier mobility $(\mu)$ was calculated using the cylinder-on-plate model that takes into consideration the electrostatic fringing effect acting on the finite number of NWs in the channel, as expressed in Equations (5) and (6) ${ }^{18,41-44}$

$$
\begin{gathered}
C_{N W}=N \frac{2 \pi \varepsilon_{o} \varepsilon_{r} L}{\cosh ^{-1}\left(\frac{r+d}{r}\right)}, \\
\mu=\frac{L^{2}}{C_{N W}} \frac{1}{V_{S D}} g_{m},
\end{gathered}
$$

where $\varepsilon_{r}$ is the $\mathrm{SiO}_{2}$ dielectric constant (3.9), $d$ is the thickness of the gate dielectric $(230 \mathrm{~nm}), V_{S D}$ is the applied S/D voltage and $g_{m}$ is the transconductance $\left(g_{m}=\frac{\partial I_{D}}{\partial V_{G}}\right)$.

The sub-threshold swing, trap density and mobility of the devices shown in Fig. 4(a) as a function of DEP alignment frequency are presented in Table I. Furthermore, similar data from a number of devices fabricated at a range of DEP signal frequencies $(30 \mathrm{~Hz}, 300 \mathrm{kHz}, 10 \mathrm{MHz}, 20 \mathrm{MHz})$ are also shown in Fig. 4(c). It is clear that SiNWs aligned at the low DEP frequencies $(30 \mathrm{~Hz}$ and $300 \mathrm{kHz})$ showed low maximum on-state current at the gate voltage $\left(\mathrm{V}_{\mathrm{G}}\right)$ of $-40 \mathrm{~V}$ (Fig. 4(d)). For NWs collected at the high frequencies $(10 \mathrm{MHz}$ and $20 \mathrm{MHz})$, the FETs showed a significantly improved performance, with two orders of magnitude higher current (per NW), 50\% lower sub-threshold swing, and with up 100 times higher mobility values, as compared to FETs with NWs collected at low frequencies (see Table I, Figs.

TABLE I. Sub-threshold swing, trap density and mobility values of the FET devices shown in Fig. 4(a).

\begin{tabular}{lccc}
\hline \hline DEP frequency $(\mathrm{Hz})$ & $\mathrm{s}-\mathrm{s}(\mathrm{V} /$ decade $)$ & $\mathrm{N}_{\text {Trap }}\left(\mathrm{cm}^{-2}\right)$ & $\mu\left(\mathrm{cm}^{2} \mathrm{~V}^{-1} \mathrm{~s}^{-1}\right)$ \\
\hline 30 & 4.1 & $1.7 \times 10^{13}$ & 0.1 \\
$300 \mathrm{k}$ & 5.0 & $2.1 \times 10^{13}$ & 0.06 \\
$10 \mathrm{M}$ & 2.1 & $8.9 \times 10^{12}$ & 7.05 \\
$20 \mathrm{M}$ & 2.6 & $1.1 \times 10^{13}$ & 8.7 \\
\hline \hline
\end{tabular}


4(c) and 4(d)). The increase in the mobility values from low to high frequencies can be attributed to several factors, including the collection of NWs with higher crystal quality and lower densities of defects and traps states, which will affect all the ability of the NWs to respond to the fast oscillating external non-uniform DEP field. ${ }^{18}$ The reduction in subthreshold slope and NW surface trap density for FET devices containing NWs collected at $20 \mathrm{MHz}$ provides further support of the selective properties of the DEP process to assemble NWs with lower trap density.

Finally, to evaluate the range of maximum on-currents that can be supported by a high quality NW FET, a device with approximately 200 nanowires in the channel was prepared by assembling NWs at $20 \mathrm{MHz}$ DEP signal frequency. This device was capable of supporting a total current of $\approx 0.65 \mathrm{~mA}\left(\mathrm{~V}_{\mathrm{G}}=-40 \mathrm{~V}, \mathrm{~V}_{\mathrm{D}}=-25 \mathrm{~V}\right)$ high on/off ratio of $10^{5}$ and the hole mobility of $16 \pm 2.3 \mathrm{~cm}^{2} \mathrm{~V}^{-1} \mathrm{~s}^{-1}$.

In summary, our results demonstrate that NWs grown in the same controllable CVD process still have significant variations in properties, as evidenced by the FET data for nanowires collected at various DEP frequencies. By analyzing the frequency spectrum obtained using the 3DEP system, we obtain a direct correlation between the properties of NWs collected at different frequencies and the corresponding FET characteristics. Thus, by altering the dielectrophoretic frequency, high or low quality NWs can be discriminated. DEP aligned NWs in the high frequency range $(10 \mathrm{MHz}$ and $20 \mathrm{MHz}$ ) showed up to 100 times higher on-current and carrier mobility values, as compared to the lower frequency range $(30 \mathrm{~Hz}, 300 \mathrm{kHz})$. We propose that beyond the immediate application for SiNWs, 3DEP analysis can be applied for efficient evaluation, the properties of a wide variety of nanostructures such as metallic and semiconducting nanowires, carbon nanotubes and nanoflakes.

M.C. thanks the A.G. Leventis Foundation for providing an Educational Grant. S.K. acknowledges the support from the U.S. Department of Commerce, National Institute of Standards and Technology under the financial assistance Award No. 70NANB16H043.

Certain commercial equipment, instruments, or materials are identified in this paper in order to specify the experimental procedure adequately. Such identification is not intended to imply recommendation or endorsement by the National Institute of Standards and Technology, nor is it intended to imply that the materials or equipment identified are necessarily the best available for the purpose.

${ }^{1}$ Y. Cui, Q. Wei, H. Park, and C. M. Lieber, Science 293(5533), 1289 (2001).

${ }^{2}$ A. Gao, N. Lu, Y. Wang, P. Dai, T. Li, X. Gao, Y. Wang, and C. Fan, Nano Lett. 12(10), 5262 (2012).

${ }^{3}$ F. Patolsky and C. M. Lieber, Mater. Today 8(4), 20 (2005).

${ }^{4}$ Y. Cui, Z. Zhong, D. Wang, W. U. Wang, and C. M. Lieber, Nano Lett. 3(2), 149 (2003).

${ }^{5}$ X. Duan, C. Niu, V. Sahi, J. Chen, J. W. Parce, S. Empedocles, and J. L. Goldman, Nature 425(6955), 274 (2003).

${ }^{6}$ G. Zheng, W. Lu, S. Jin, and C. M. Lieber, Adv. Mater. 16(21), 1890 (2004).

${ }^{7}$ M. S. Gudiksen, L. J. Lauhon, J. Wang, D. C. Smith, and C. M. Lieber, Nature 415(6872), 617 (2002).
${ }^{8}$ X. Duan, Y. Huang, R. Agarwal, and C. M. Lieber, Nature 421(6920), 241 (2003).

${ }^{9}$ M. Chen, H. Y. Yu, N. Singh, Y. Sun, N. S. Shen, X. Yuan, G.-Q. Lo, and D.-L. Kwong, IEEE Electron Device Lett. 30(8), 879 (2009).

${ }^{10}$ Y. Sun, H. Yu, N. Singh, K. Leong, E. Gnani, G. Baccarani, G. Lo, and D. Kwong, IEEE Trans. Electron Devices 58(5), 1329 (2011).

${ }^{11}$ B. Yang, K. Buddharaju, S. Teo, N. Singh, G. Lo, and D. Kwong, IEEE Electron Device Lett. 29(7), 791 (2008).

${ }^{12}$ J. Song, J. Zhou, and Z. L. Wang, Nano Lett. 6(8), 1656 (2006).

${ }^{13}$ B. Tian, T. J. Kempa, and C. M. Lieber, Chem. Soc. Rev. 38(1), 16 (2009).

${ }^{14}$ A. Tao, F. Kim, C. Hess, J. Goldberger, R. He, Y. Sun, Y. Xia, and P. Yang, Nano Lett. 3(9), 1229 (2003).

${ }^{15}$ G. Yu, X. Li, C. M. Lieber, and A. Cao, J. Mater. Chem. 18(7), 728 (2008).

${ }^{16}$ Y. Huang, X. Duan, Q. Wei, and C. M. Lieber, Science 291(5504), 630 (2001).

${ }^{17}$ K. Heo, E. Cho, J.-E. Yang, M.-H. Kim, M. Lee, B. Y. Lee, S. G. Kwon, M.-S. Lee, M.-H. Jo, and H.-J. Choi, Nano Lett. 8(12), 4523 (2008).

${ }^{18}$ M. Constantinou, G. P. Rigas, F. Castro, V. Stolojan, K. Hoettges, M. Hughes, E. Adkins, B. A. Korgel, and M. Shkunov, ACS Nano 10(4), 4384 (2016)

${ }^{19}$ M. P. Hughes, Nanoelectromechanics in Engineering and Biology (CRC Press, 2002).

${ }^{20}$ H. Mulhall, F. Labeed, B. Kazmi, D. Costea, M. Hughes, and M. Lewis, Anal. Bioanal. Chem. 401(8), 2455 (2011).

${ }^{21}$ K. Graham, H. Mulhall, F. Labeed, M. Lewis, K. Hoettges, N. Kalavrezos, J. McCaul, C. Liew, S. Porter, and S. Fedele, Analyst 140(15), 5198 (2015).

${ }^{22}$ H. J. Mulhall, M. P. Hughes, B. Kazmi, M. P. Lewis, and F. H. Labeed, Biochim. Biophys. Acta, Gen. Subj. 1830(11), 5136 (2013).

${ }^{23}$ X. Liang, K. Graham, A. Johannessen, D. Costea, and F. Labeed, Integr. Biol. 6(5), 545 (2014)

${ }^{24}$ K. F. Hoettges, Y. Hübner, L. M. Broche, S. L. Ogin, G. E. Kass, and M. P. Hughes, Anal. Chem. 80(6), 2063 (2008).

${ }^{25}$ M. Dimaki and P. Bøggild, Nanotechnology 15(8), 1095 (2004).

${ }^{26} \mathrm{E}$. M. Freer, O. Grachev, X. Duan, S. Martin, and D. P. Stumbo, Nat. Nanotechnol. 5(7), 525 (2010).

${ }^{27}$ T. B. Jones and T. B. Jones, Electromechanics of Particles (Cambridge University Press, 2005).

${ }^{28}$ H. Morgan and N. G. Green, J. Electrost. 42(3), 279 (1997).

${ }^{29}$ S. Raychaudhuri, S. A. Dayeh, D. Wang, and E. T. Yu, Nano Lett. 9(6), 2260 (2009).

${ }^{30}$ Z. Wang, M. Kroener, and P. Woias, Sens. Actuators, A 188, 417 (2012).

${ }^{31}$ L. M. Broche, F. H. Labeed, and M. P. Hughes, Phys. Med. Biol. 50(10), 2267 (2005).

${ }^{32}$ Y. Wang, K.-K. Lew, T.-T. Ho, L. Pan, S. W. Novak, E. C. Dickey, J. M. Redwing, and T. S. Mayer, Nano Lett. 5(11), 2139 (2005).

${ }^{33}$ S. M. Sze and K. K. Ng, Physics of Semiconductor Devices (John Wiley \& Sons, 2006).

${ }^{34}$ E. H. Williams, J. A. Schreifels, M. V. Rao, A. V. Davydov, V. P. Oleshko, N. J. Lin, K. L. Steffens, S. Krylyuk, K. A. Bertness, and A. K. Manocchi, J. Mater. Res. 28(01), 68 (2013).

${ }^{35}$ V. P. Oleshko, T. Lam, D. Ruzmetov, P. Haney, H. J. Lezec, A. V. Davydov, S. Krylyuk, J. Cumings, and A. A. Talin, Nanoscale 6(20), 11756 (2014).

${ }^{36}$ X. H. Liu, J. W. Wang, S. Huang, F. Fan, X. Huang, Y. Liu, S. Krylyuk, J. Yoo, S. A. Dayeh, and A. V. Davydov, Nat. Nanotechnol. 7(11), 749 (2012).

${ }^{37}$ Y. Paska and H. Haick, ACS Appl. Mater. Interfaces 4(5), 2604 (2012).

${ }^{38}$ Y. Paska, T. Stelzner, O. Assad, U. Tisch, S. Christiansen, and H. Haick, ACS Nano 6(1), 335 (2012).

${ }^{39}$ M. McDowell, I. Hill, J. McDermott, S. Bernasek, and J. Schwartz, Appl. Phys. Lett. 88(7), 073505 (2006).

${ }^{40}$ M.-H. Yoon, C. Kim, A. Facchetti, and T. J. Marks, J. Am. Chem. Soc. 128(39), 12851-12869 (2006).

${ }^{41}$ O. Wunnicke, Appl. Phys. Lett. 89(8), 083102 (2006).

${ }^{42}$ S. Ramo, J. R. Whinnery, and T. Van Duzer, Fields and Waves in Communication Electronics (John Wiley \& Sons, 2007).

${ }^{43}$ C. Opoku, K. Hoettges, M. Hughes, V. Stolojan, S. Silva, and M. Shkunov, Nanotechnology 24(40), 405203 (2013).

${ }^{44}$ R. F. Harrington, Introduction to Electromagnetic Engineering (Courier Corporation, 2003). 